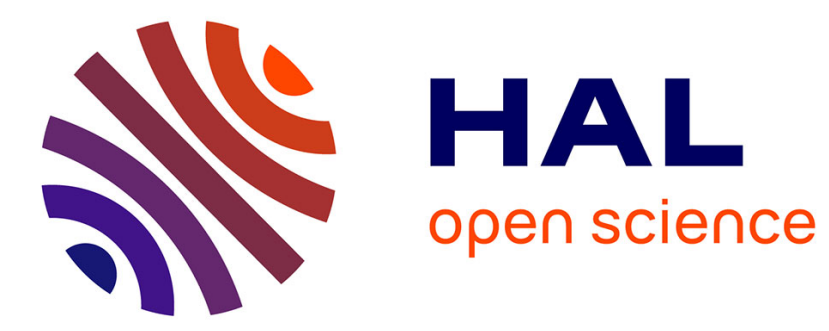

\title{
Perturbation-iteration methods for large perturbations in quantum mechanics
}

Ph. Durand

\section{To cite this version:}

Ph. Durand. Perturbation-iteration methods for large perturbations in quantum mechanics. Journal de Physique Lettres, 1982, 43 (13), pp.461-469. 10.1051/jphyslet:019820043013046100 . jpa00232077

\section{HAL Id: jpa-00232077 https://hal.science/jpa-00232077}

Submitted on 1 Jan 1982

HAL is a multi-disciplinary open access archive for the deposit and dissemination of scientific research documents, whether they are published or not. The documents may come from teaching and research institutions in France or abroad, or from public or private research centers.
L'archive ouverte pluridisciplinaire HAL, est destinée au dépôt et à la diffusion de documents scientifiques de niveau recherche, publiés ou non, émanant des établissements d'enseignement et de recherche français ou étrangers, des laboratoires publics ou privés. 


\title{
LE JOURNAL DE PHYSIQUE-LETTRES
}

J. Physique - LETTRES 43 (1982) L-461 - L-469

1er JUILLET 1982, PAGE L-461

Classification

Physics Abstracts

03.65

\section{Perturbation-iteration methods for large perturbations in quantum mechanics}

\author{
Ph. Durand \\ Laboratoire de Physique Quantique (*), Université Paul Sabatier, \\ 118, route de Narbonne, 31062 Toulouse Cedex, France
}

(Reçu le 16 novembre 1981, révisé le 27 avril 1982, accepté le 10 mai 1982)

\begin{abstract}
Résumé - L'introduction d'un opérateur d'onde réduit $X$ permet de présenter simplement la méthode usuelle de Rayleigh-Schrödinger. Au-delà de ce schéma et pour de fortes perturbations, nous proposons de déterminer $X$ par de nouvelles méthodes itératives à convergence linéaire, quadratique ou quasi quadratique. L'étude d'un système à deux états montre les principales caractéristiques de ces méthodes. La détermination des plus bas états excités neutres d'une molécule en présence d'états ioniques intrus illustre les possibilités nouvelles offertes par ces méthodes.
\end{abstract}

\begin{abstract}
The introduction of a reduced wave operator $X$ allows us to present in a systematic and transparent way the usual Rayleigh-Schrödinger method. Beyond this scheme and for large perturbations, $X$ is determined by new iterative methods which converge linearly, quadratically or quasiquadratically. The study of a two-state system exhibits the main characteristics of these methods. The new possibilities afforded by these methods are illustrated by determining the spectrum of the neutral states of a molecule in presence of ionic intruder states.
\end{abstract}

The standard Rayleigh-Schrödinger perturbation method is very efficient for small perturbations. For large perturbations and degenerate systems many difficulties arise. Most often the perturbation series for wave functions and energies diverge. Although powerful methods for improving the convergences were previously derived, such as for example summation techniques and Padé approximants, the problem of large perturbations is still an open problem [1]. Perhaps the most typical situation can be found in Many-Body-Perturbation Theories (MBPT) where diagrammatic methods and partial summations to all orders cannot be considered as the final stage of perturbation methods [2]. It is to be noticed that these methods, strangely enough, were

$\left(^{*}\right)$ E.R.A. du C.N.R.S. no 821 . 
developed in Physics outside the field of the most standard and currently used methods of numerical analysis. The aim of this letter is to show that, in fact, there are powerful and systematic algebraic methods for handling large perturbations. All these methods are ultimately based on implicit polynomial or rational function expansions with well defined linear, quadratic or quasiquadratic convergences.

1. Non-degenerate state. - We consider an exact Hamiltonian

$$
H=H_{0}+V
$$

where $H_{0}$ is the unperturbed Hamiltonian and $V$ is a perturbation. The eigenstates of $H_{0}$ are partitioned into two subspaces $\Omega_{0}$ and its orthogonal complement $\Omega_{0}^{\perp}$. Let us first suppose that $\Omega_{0}$ is spanned by a unique non-degenerate state noted $|0\rangle$. One can pass from the unperturbed state $|0\rangle$ to the exact solution $|\Psi\rangle=U|0\rangle$ by means of the wave operator $U$. Assuming the intermediate normalization, the wave operator obeys the Bloch equation [3]

$$
U=P_{0}+\frac{Q_{0}}{a}(V U-U V U)
$$

where $P_{0}$ and $Q_{0}=1-P_{0}$ are the projectors onto $\Omega_{0}$ and $\Omega_{0}^{\perp}$ respectively. $\frac{Q_{0}}{a} \equiv \frac{Q_{0}}{E_{0}-H_{0}}$ and $E_{0}$ is the energy of the unperturbed state. Multiplying both sides of (2) by $Q_{0}$ and introducing the reduced wave operator $X=Q_{0} U P_{0}$ leads to the equation

$$
X=\frac{Q_{0}}{a}(1-X) V(1+X) P_{0} .
$$

The determination of $X$ by the Rayleigh-Schrödinger (RS) method is obtained by expanding $X$ in orders of perturbation : $X=X^{(1)}+X^{(2)}+\cdots$. Substituting this development into (3) and identifying both sides of this expression lead to the RS expansion $[3,4]$.

$$
\begin{aligned}
& X^{(1)}=\frac{Q_{0}}{a} V P_{0} \\
& X^{(2)}=\frac{Q_{0}}{a}\left[V, X^{(1)}\right] P_{0} \\
& \ldots \ldots \ldots \ldots \ldots \ldots \\
& X^{(n)}=\frac{Q_{0}}{a}\left\{\left[V, X^{(n-1)}\right]-\sum_{k=1}^{n-2} X^{(k)} V X^{(n-k-1)}\right\} P_{0}
\end{aligned}
$$

This expansion appears as a complicated recurrent structure with rather poor convergence properties $[1,2,5]$.

Instead of solving (3) by (4) we propose to determine $X$ by methods which converge linearly or quadratically. The introduction of the notation

$$
f(X) \equiv \frac{Q_{0}}{a}(1-X) V(1+X) \text { and } F(X) \equiv X-f(X)
$$

allows us to write (3) in the form :

$$
X=f(X) \text { or } \quad F(X)=0 .
$$


Although (6) is an operator equation, notation (6) suggests that it could be solved by iterative procedures similar to those used for the resolution of algebraic equations [6]. The simplest procedure is given by

$$
X^{(n)}=f\left(X^{(n-1)}\right)
$$

or by introducing the increment $\Delta X^{(n)}=X^{(n)}-X^{(n-1)}$ at the $n$-th iteration :

$$
\Delta X^{(n)}=-F\left(X^{(n-1)}\right) \quad \text { (linear method). }
$$

(7) or (8) define an iterative procedure for determining $X$, simpler than the RS expansion, which converges linearly for small perturbations. This procedure leads to an implicit polynomial expansion of $X$ in orders of perturbation with a strong mixing of the orders of perturbation at each iteration (see below the two-state system). Although this linear method converges better than the RS expansion, it still diverges for large perturbations where it must be replaced by a quadrically converging procedure :

$$
\Delta X^{(n)}=-C \cdot F\left(X^{(n-1)}\right) \quad \text { (quadratic method). }
$$

$C$ is a linear operator or superoperator [7] acting in the vector space of all the transition operators $X_{i}=|i\rangle\langle 0|$ between $|0\rangle$ and $|i\rangle \in \Omega_{0}^{\perp} . C=A^{-1}$ is called the convergence operator. It is the inverse of an operator $A$ defined by

and

$$
\begin{aligned}
A . X_{i} & =\sum_{j} X_{j} \cdot(j|A| i) \\
(i|A| i) & =1+\left\langle i\left|\frac{Q_{0}}{a}\right| i\right\rangle\langle 0|V(1+X)| 0\rangle-\left\langle i\left|\frac{Q_{0}}{a}(1-X) V\right| i\right\rangle \\
(i|A| j) & =-\left\langle i\left|\frac{Q_{0}}{a}(1-X) V\right| j\right\rangle \text { for } j \neq i .
\end{aligned}
$$

The iterative procedure (8) to (12) is essentially a generalized Newton-Raphson method for the solution of equation (6). This procedure converges quadratically and corresponds implicitly to a rational function expansion with two polynomials of increasing orders of perturbation (see below the two-state system). It must be noted that for small perturbations $C$ goes toward the identity operator and that for large perturbations the convergence properties of the method rely on the departure of $C$ from the identity operator. From a computational point of view, the use of an exact quadratic procedure would imply the inversion of large matrices. In fact, many approximations can be made on $C=A^{-1}$ that lead to various quasi-quadratic procedures [8].

2. Degenerate or quasi-degenerate states. - Let us now consider $n$ degenerate or quasi-degenerate states denoted $|a\rangle$ spanning a $n$-dimension model space $\Omega_{0}$. In this case the generalized Bloch equation [9] leads to a generalization of (3) :

$$
X=\sum_{a} \frac{Q_{0}}{a}(1-X) V(1+X) P_{a} .
$$

$P_{a}=|a\rangle\langle a|$ is the projector onto the unperturbed states of the $n$-dimension model space ; $P_{0}=\sum_{a} P_{a}$ and $\frac{Q_{0}}{a} \equiv \frac{Q_{0}}{E_{a}^{0}-H_{0}}$. Equation (13) can be solved by iterative methods similar to those presented for a non-degenerate state. The details of these methods will be given in subsequent papers. The derivation of $X$ leads to states $\left|\phi_{a}\right\rangle=(1+X)|a\rangle$ which are linear combi- 
nations of the true solutions. These solutions can be obtained immediately through the diagonalization of an $n$-dimension effective Hamiltonian such as those previously proposed by Kato [5], Bloch [3] and des Cloizeaux [10]. The knowledge of the $\phi_{a}$ 's is also a good starting point for determining various useful effective Hamiltonians and transferable interactions or parameters [11].

2.1 TWO-STATE SYSTEM. - In the basis of the two unperturbed states $|0\rangle$ and $|1\rangle$ the exact Hamiltonian is given by

$$
H=|1\rangle\langle 1|-\lambda(|0\rangle\langle 1|+| 1\rangle\langle 0|) .
$$

In this simple case, the operator equation (6) reduces to the ordinary algebraic equation :

$$
x=\lambda\left(1-x^{2}\right)
$$

with the exact useful solution

$$
x=\frac{\sqrt{1+4 \lambda^{2}}-1}{2 \lambda}
$$

Recurrence (8) becomes

$$
\Delta x_{\text {linear }}^{(n)}=-\left[x^{(n)}-\lambda\left[1-\left(x^{(n-1)}\right)^{2}\right]\right]
$$

and equation (9) reduces to the recurrence of the ordinary Newton-Raphson method :

$$
\Delta x_{\text {quadratic }}^{(n)}=-C \cdot \Delta x_{\text {linear }}^{(n)}
$$

where $C=\frac{1}{1+2 \lambda x^{(n-1)}}$. The quasi-quadratic method is characterized by a constant value $C=\frac{1}{1+2 \lambda^{2}}$ obtained by approximating $x^{(n-1)}$ in $C$ by its first order Rayleigh-Schrödinger value $x^{(1)}=\lambda$.

The explicit expansions of $x$ are :

Rayleigh-Schrödinger

$$
\begin{aligned}
& x^{(1)}=\lambda \\
& x^{(3)}=\lambda-\lambda^{3} \\
& x^{(5)}=\lambda-\lambda^{3}+2 \lambda^{5}
\end{aligned}
$$

Quadratic method (Newton-Raphson)

$$
\begin{aligned}
& x^{(1)}=\lambda \\
& x^{(2)}=\frac{\lambda+\lambda^{3}}{1+2 \lambda^{2}} \\
& x^{(3)}=\frac{\lambda+5 \lambda^{3}+6 \lambda^{5}+\lambda^{7}}{1+6 \lambda^{2}+10 \lambda^{4}+4 \lambda^{6}}
\end{aligned}
$$

\section{Linear method}

$$
x^{(1)}=\lambda
$$$$
x^{(2)}=\lambda-\lambda^{3}
$$$$
x^{(3)}=\lambda-\lambda^{3}+2 \lambda^{5}-\lambda^{7}
$$

Figures 1 to 4 display the convergence properties of these methods as functions of parameter $\lambda$. 


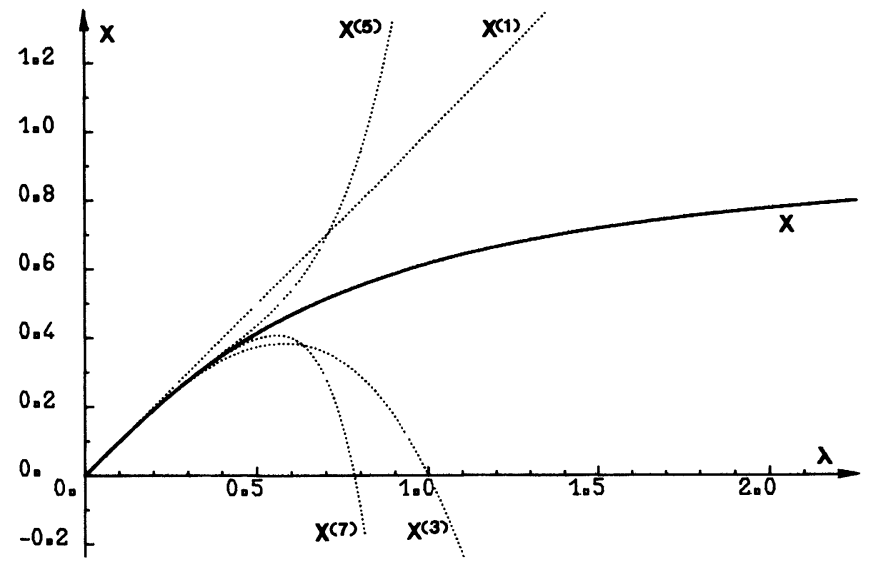

Fig. 1. - The Rayleigh-Schrödinger method : — exact result (Eq. (17)); . . . . expansion of $x$ to orders $1,3,5,7$ of perturbation.

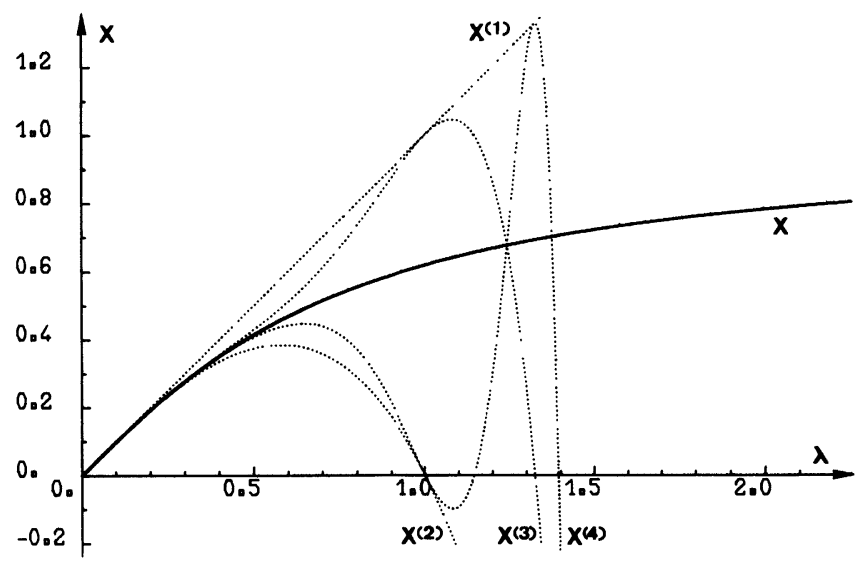

Fig. 2. - Linear method : — exact result (Eq. (17)) ; . . . approximate values of $x$ for iterations 1, 2, 3 (Eq. (18)).

The Rayleigh-Schrödinger method strongly diverges beyond $\lambda \simeq 0.5$ with oscillations in the form of a bent palm-tree. The linear method converges up to $\lambda=\sqrt{3} / 2=0.87$, oscillates for $\sqrt{3} / 2<\lambda<1$ and diverges beyond $\lambda=1$. A comparison between figures 1 and 2 shows that the Rayleigh-Schrödinger can be considered as an approximate or quasi-linear method with its characteristic oscillating $x$ values at the various iterations.

Figures 3 and 4 show very similar strong monotonous convergence properties. In particular the exact quadratic method converges for any value of $\lambda \in[0, \infty]$. The main interest of this twostate system is that it gives a nice illustration of convergence properties which remains essentially significant for real systems containing a large number of unperturbed states.

2.2 GROUND AND FIRST NEUTRAL EXCITED STATES OF A TETRAHEDRAL MOlECULE. - One can classify the ground and first excited states of a conjugated molecule or of a metallic cluster of atoms in mainly neutral and mainly ionic states. This terminology refers to the content of the wave functions with respect to a basis set of Slater determinants. In the framework of the valence 


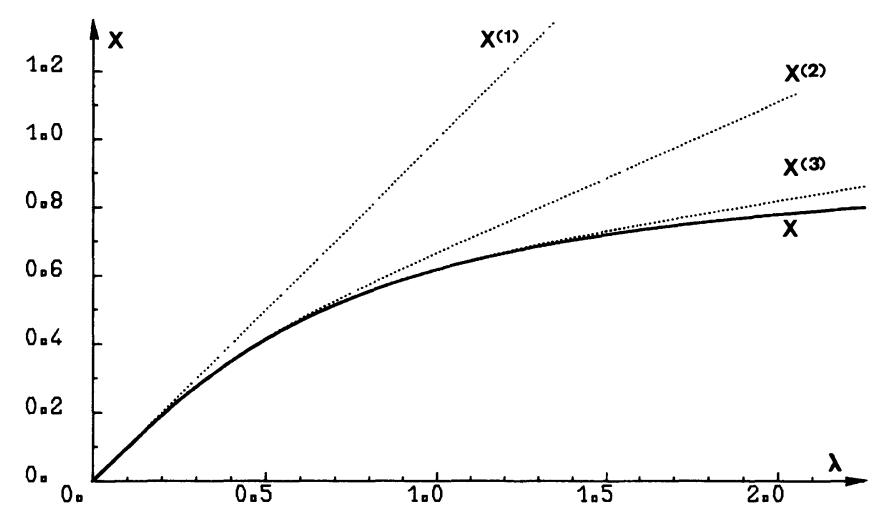

Fig. 3. - Quadratic method : — exact result (Eq. (17)); . . . . . approximate values of $x$ for iterations 1, 2, 3 (Eq. (18)).

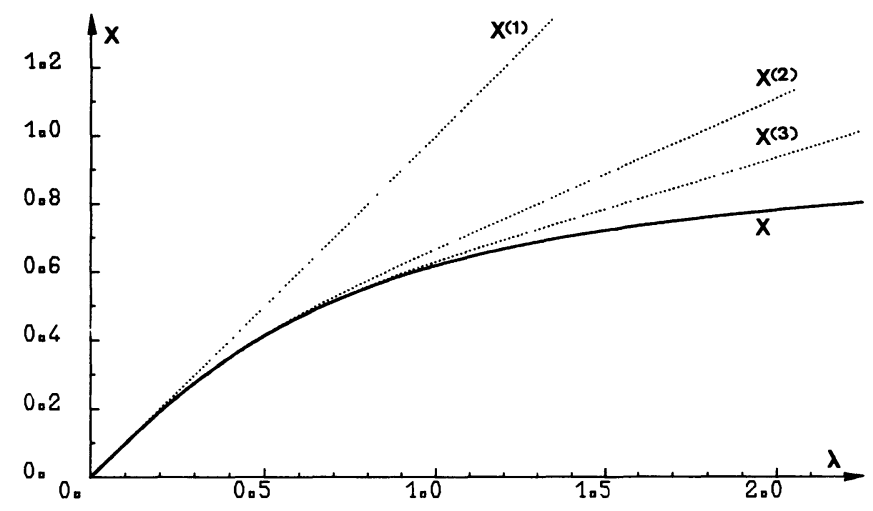

Fig. 4. - Quasi-quadratic method : exact result (Eq. (17)); . . . . a approximate values of $x$ for iterations 1, 2, 3 (Eq. (18)).

bond (VB) theory these determinants can be divided into neutral and ionic determinants. The neutral determinants with one electron per atom have the lowest energies. The monoionic determinants which are characterized by the transfer of an electron from an atom to a neighbour atom have higher energies. Then appear the diionic determinants etc... The classification of the basis determinants by increasing energies remains essentially unmodified for the exact solutions : the ground and first neutral excited states have small components on the ionic determinants. At higher energies the weight of the ionic contributions becomes more and more important. Note that there is no clear frontier between the mainly neutral and mainly ionic states. The determination of the neutral states alone and of the corresponding effective interactions between the electrons is a difficult task for two reasons. First, these states are highly degenerate even for systems containing a small number of atoms. Second, the highest states are mixed with mainly ionic states, especially for compact structures which are stabilized by the Madelung field. These states which can be considered as intruder states lead to well known convergence difficulties for deriving the effective interactions between the particles [12].

For demonstrating the efficiency of our methods in a case where standard methods fail, we have chosen a four-atom four-electron tetrahedral model molecule. One and two-electron interactions are described within the framework of a Hubbard Hamiltonian [13] with parameters 
from reference [14]. All solutions with $S_{z}=0$ arise from a thirty-six unperturbed basis set made up of six neutral, twenty-four monoionic and six diionic determinants (Fig. 5).

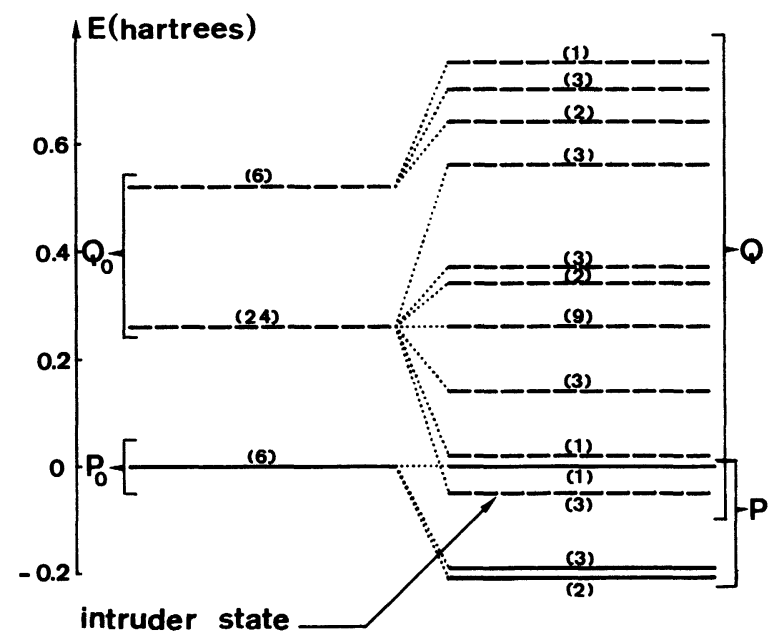

Fig. 5. - Ground and excited states $\left(S_{z}=0\right)$ of a four-electron tetrahedral molecule. $P_{0}$ and $Q_{0}$ span the unperturbed neutral (- ${ }^{-}$and ionic (-...-.) subspaces. The degeneracies of the unperturbed (left) and perturbed (right) states are given in parentheses.

The Bloch and des Cloizeaux effective Hamiltonians were easily determined in seven iterations leading to energies with six exact figures given in table $\mathrm{I}$.

Table I. - Energies in Hartrees (1 Hartree = 27.2 eV) of a four-electron molecule described by a Hubbard Hamiltonian. Degeneracies are given in parentheses.

Unperturbed energies

$$
0.513481
$$

$0.256740(24)$

$0.000000 \quad(6)$
Exact energies

$\begin{array}{rr}0.143860(3) & 0.752282(1) \\ 0.017939(1) & 0.702501(3) \\ -0.000000(1) & 0.636551(2) \\ -0.045160(3) & 0.558640(3) \\ -0.189020(3) & 0.369620(3) \\ -0.208954(2) & 0.342625(2) \\ & 0.256740(9)\end{array}$

It is particularly interesting to note that our procedure converges within the subspace of the neutral states, avoiding the triply degenerate ionic intruder state with energy $E=-0.045$ Hartree. The efficiency of our method can be understood by considering that the solution of Bloch-type equations $F(X)=0$ can also be obtained by minimizing

$$
(F(X) \mid F(X)) \equiv \operatorname{Tr}\left(F^{+}(X) F(X)\right) .
$$

It means that our procedure is implicitly based on a variational principle on the wave functions whereas most of the standard methods of resolution of the Schrödinger equation are based on the Rayleigh-Ritz variational principle on the energies. From the different nature of these minimum principles we find that our method is stable with respect to the physical content of the effec- 
tive Hamiltonian (the neutral states in our example) : we can easily pass from the unperturbed subspace with projector $P_{0}$ to the nearest exact subspace with projector $P$.

For the tetrahedral molecule, the Rayleigh-Schrödinger method diverges. The calculations have also been performed by the Davidson method [15] which we consider as one of the most powerful method for deriving sequentially eigensolutions of large matrices. The iterative procedure was initiated from zero-order eigenvectors in the form of a linear combination of the six neutral states. The method was applied by requiring various precisions on the energies $\left(10^{-3}\right.$, $10^{-6}$ and $10^{-9}$ a.u.). In all the cases the method failed to reproduce twice the energy of the degenerate ground state. It gave four times the value -0.189 a.u. which is triply degenerate. But the most difficult problem to deal with was the instability of the results : the sequence of the eigenstates depends on the required precision on the energies. For the sixth eigenvalue, for example, the method led to the highest neutral state for a precision of 0.001 a.u. whereas a precision of $10^{-9}$ a.u. led to the ionic intruder state. Clearly the sequential determination of eigensolutions does not give any guarantee for remaining stable within the subspace of the neutral states and thus for determining the significant parameters of the effective Hamiltonian. Some convergence difficulties were also found but they could be overcome by improving the initial solution.

Other test calculations were also performed with our method on various electron and spin model Hamiltonians. In all the cases good convergences were obtained even for very degenerate systems.

2.3 DisCuSSION AND CONCLUSIONS. - In contrast with other methods which try to determine either directly the energies [16] or both the energies and the wave functions $[15,17,18]$ the general methodology presented in this letter leads to a direct determination of the wave functions.

The direct determination of energies would imply the resolution of the equation

$$
F(E) \equiv E-f(E)=0 \quad \text { where } \quad f(E)=\left\langle 0\left|H+V \frac{Q_{0}}{E-H} V\right| 0\right\rangle
$$

Unfortunately the determination of $\frac{Q_{0}}{E-H}$ requires about $N^{3}$ multiplications for an $N$-dimension problem. In fact the most efficient standard methods determine simultaneously both the energies and the wave functions and require $N^{2}$ multiplications per eigenvalue at each iteration. They are most often based on the Rayleigh-Ritz variational principle and there is no guarantee for remaining stable within the subspace of physical interest especially in presence of intruder states. Finally the method presented in this letter combines the computational efficiency $\left(n N^{2}\right.$ multiplication at each iteration for an $n$-dimension effective Hamiltonian) and good convergence properties for highly degenerate system and/or in presence of intruder states. Its efficiency has been checked for matrices up to $N=1000$ for deriving spin effective Hamiltonians of order up to $n=20$.

A first comparison between the existing methods and ours suggests that it could most often be easier in quantum mechanics to determine first the wave functions and then to calculate the energies which depend quadratically upon the wave functions.

Acknowledgments. - The author is grateful to J. P. Malrieu and D. Maynau for helpful discussions.

\section{References}

[1] For molecules the present state of the art can be found in the proceedings of the NRCC conference, Seattle, USA, July, 1981. 
[2] For a review and examples of perturbation methods applied to nuclei see : « Effective interactions and operators in nuclei ", B. R. Barrett, ed., Lectures Notes in Physics, Vol. 40 (Springer Verlag) 1975 , p. 1.

[3] Bloch, C., Nucl. Phys. 6 (1958) 329.

[4] A similar but different $X$ operator has recently been introduced by SHAvitT, I. and ReDMon, L. T., in J. Chem. Phys. 73 (1980) 5711. The exact relationship between the $X$ operator of Shavitt and Redmon denoted $X_{\mathrm{SR}}$ and the operator $X$ defined in this paper is : $X=Q_{0} X_{\mathrm{SR}} P_{0}, X_{\mathrm{SR}}=X+X^{+}$.

[5] Kato, T., Prog. Theor. Phys. 4 (1949) 514 ; 5 (1950) 95, 207.

[6] Durand, E., Solutions numériques des équations algébriques, Tome I (Masson et Cie, Paris) 1960, p. 31.

[7] Primas, H., Helv. Phys. Acta 34 (1961) 331 ; Rev. Mod. Phys. 35 (1963) 710.

[8] Durand, Ph., to be published.

[9] J $\phi$ Rgensen, F., Mol. Phys. 29 (1975) 1137.

[10] Des Cloizeaux, J., Nucl. Phys. 20 (1960) 321.

[11] Nicolas, G. and Durand, Ph., J. Chem. Phys. 72 (1980) 453.

[12] Ellis, P. J. and Osnes, E., Rev. Mod. Phys. 49 (1977) 777.

[13] Hubbard, J., Proc. R. Soc. A 276 (1963) 238.

[14] Malrieu, J. P. and Maynau, D., J. Am. Chem. Soc. (1982), in press.

[15] Davidson, E. R., J. Comput. Phys. 17 (1975) 87.

[16] Löwdin, P. O., J. Math. Phys. 3 (1962) 969.

[17] Jeziorski, B. and Kolos, W., Int. J. Quant. Chem., Vol. XII, Suppl. 1 (1977) 127.

[18] Kutzelnigg, W., Chem. Phys. Lett. 83 (1981) 156 and J. Chem. Phys., to be published. 